\title{
NUEVOS REGISTROS DE PROTOBRANCHIA (MOLLUSCA: BIVALVIA) PARA EL MAR PERUANO
}

\section{NEW RECORDS OF PROTOBRANCHIA (MOLLUSCA: BIVALVIA) IN THE PERUVIAN SEA}

\author{
Carlos Paredes y Franz Cardoso*
}

\section{RESUMEN}

Se registra por primer vez en aguas peruanas las siguientes especies de Bivalvia: Nucula (Nucula) iphigenia Dall, 1908; Ennucula cardara (Dall, 1916); Nuculana (Nuculana) extenuata (Dall, 1897); Orthoyoldia panamensis (Dall, 1908). Estos bivalvos fueron colectados en la plataforma y talud continental hasta profundidades de 864 metros

Palabras clave: Mollusca, Protobranchia, nuevos registros, Perú.

\begin{abstract}
The following species of Bivalvia have been recorded for the first time for Peruvian waters: Nucula (Nucula) iphigenia Dall, 1908; Ennucula cardara (Dall, 1916); Nuculana (Nuculana) extenuata (Dall, 1897); Orthoyoldia panamensis (Dall, 1908). These bivalves were collected in the platform and continental slope down to depths of 864 meters.
\end{abstract}

Key words: Mollusca, Protobranchia, new records, Peru.

\section{INTRODUCCIÓN}

Se han realizado numerosos estudios taxonómicos y ecológicos de moluscos litorales en el Perú (e. g., Vegas-Vélez, 1968; Peña, 1971; Paredes, 1974; Paredes et al., 1999 b), pero son escasos los trabajos referentes a moluscos de la plataforma y del talud continental, con excepción del trabajo de Valdivieso (1984). En esta oportunidad se reporta por primera vez para el mar peruano cuatro especies de bivalvos Protobranchia.

\section{MATERIAL Y MÉTODOS}

Los ejemplares fueron colectados durante el Crucero BIC HUMBOLTD 9607-08 (del 18 de julio al 06 de agosto de 1996), dentro del programa de localización, distribución y

* Laboratorio de Biologia y Sislemálica de Inver. tebrados Marinos, Facultad de Ciencias Biológicas, UNMSM, Apdo. 11-0058, Lima 11, Perú: Email: d190043@unmsm.edu.pe. concentración de langostinos rojos de profundidad, abarcando el área entre Huarmey y Puerto Pizarro, sobre el talud continental entre 368 y $1021 \mathrm{~m}$ de profundidad (Kameya et al., 1997). El material fue fijado con formol al $7 \%$ neutralizado con bórax y conservado en alcohol etílico al $70 \%$. Para la determina. ción se utilizó la bibliografía disponible, y para el ordenamiento sistemático se siguió a Coan et al. (2000). Este material está depositado en la colección del Laboratorio de Biología y Sistemática de Invertebrados Marinos de la Facultad de Ciencias Biológicas, Universidad Nacional Mayor de San Marcos.

\section{RESULTADOS}

Orden NUCULOIDA Superfamilia NUCULOIDEA Familia Nuculidae Gray, 1824 Nucula (Nucula) iphigenia Dall, 1896 (Figura 1) 


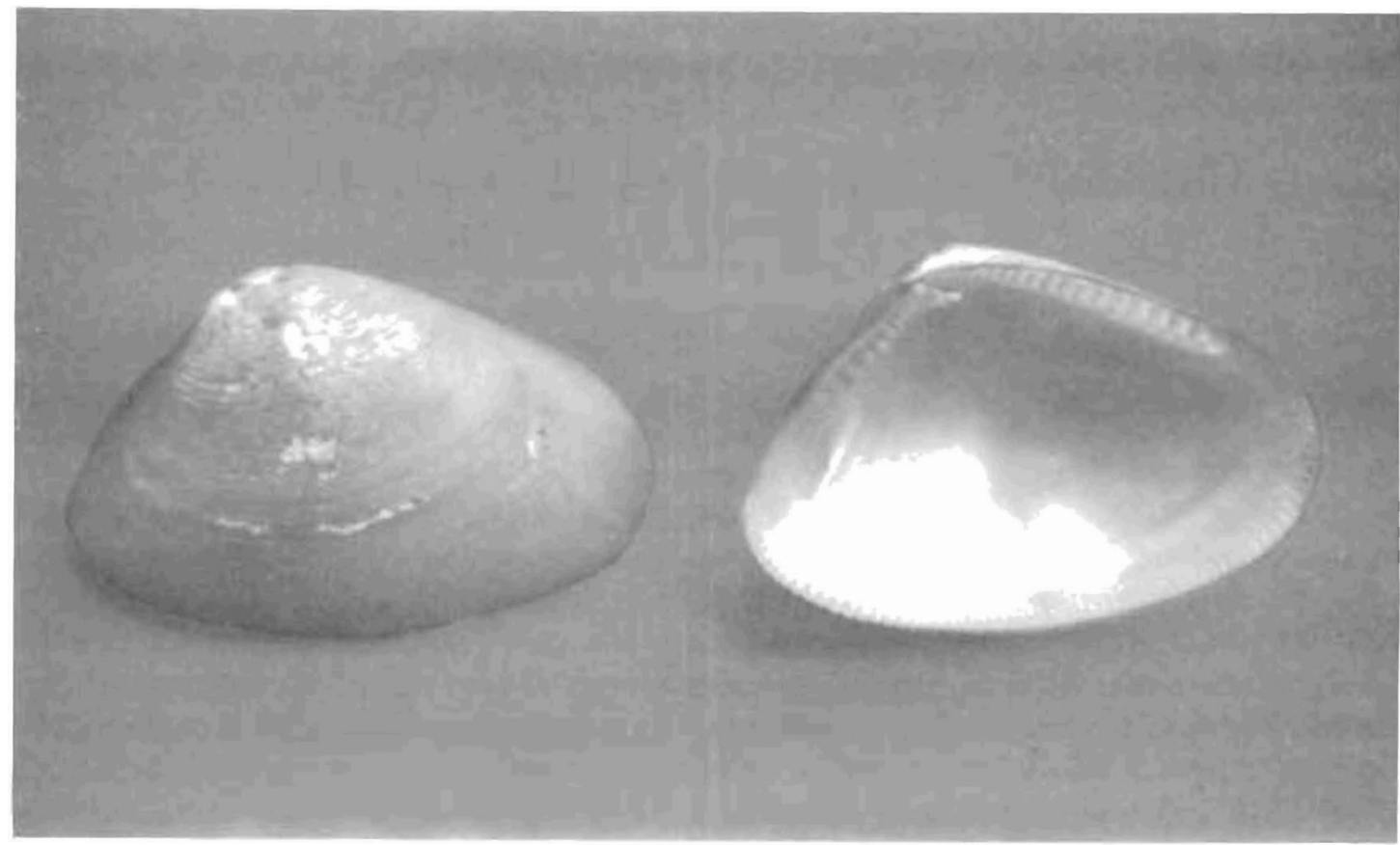

Figura 1. Nucula (Nucula) iphigenia, longitud 19,0 mm.

Nucula (N.) iphigenia Dall, 1896: 15;

Olsson, 1961: 55; Keen, 1971: 26, Fig. 6;

Bernard, 1983: 9.

Nucula (N.) iphigenia azulensis Olsson, 1942: 24, pl. 4, Fig. 2, 5, 7; Bernard, 1983: 55 , pl. 2, Fig. 9, 9 a.

Conchilla casi triangular con el extremo posterior redondeado; margen interno crenulado; periostraco amarillento e interior nacarado; escultura finamente cancelada más suave en el extremo posterior; charnela con 11 dientes anteriores y 22 posteriores. Longitud $19,0 \mathrm{~mm}$.

Distribución geográfica: Bahía de Panamá a $474 \mathrm{~m}$ (Keen, 1971).

Nueva localidad: $03^{\circ} 48,45^{\prime} \mathrm{S} ; 81^{\circ} 10,79^{\prime}$ W.

Hábitat: Fondo blando a $404 \mathrm{~m}$.

Material examinado: 1 lote, 1 ejemplar, 2807-96.

Comentarios: Bernard (1983) la reporta para el Pleistoceno a $\operatorname{los} 08^{\circ} \mathrm{N}$.

\section{Ennucula cardara (Dall, 1916)}

(Figura 2)

Nucula (Leionucula) cardara Dall, 1916: 9; Bernard, 1983: 10.

Ennucula cardara (Dall, 1916); Skoglund, 1991: 3; Coan etal., 2000: 76, pl. 4.

Conchilla triangular a oval: periostraco pardo oliváceo y brillante; prodisoconcha prominente; estrías concéntricas bien definidas e interior nacarado; borde intemo de la conchilla liso; charnela con 11 dientes anteriores y 21 posteriores. Longitud, $17,7 \mathrm{~mm}$.

Distribución geográfica: Cabo Flattery Washington a Cabo San Lucas, Baja California Sur, en 1600-2,600 m (Coan et al., 2000).

Nueva localidad: $03^{\circ} 57,37^{\circ} \mathrm{S} ; 81^{\circ} 15,24^{\prime}$ W.

Hábitat: Fondo blando, a $551 \mathrm{~m}$.

Material examinado: I Lote, 2 ejemplares (sólo valvas), 26-07-96. 


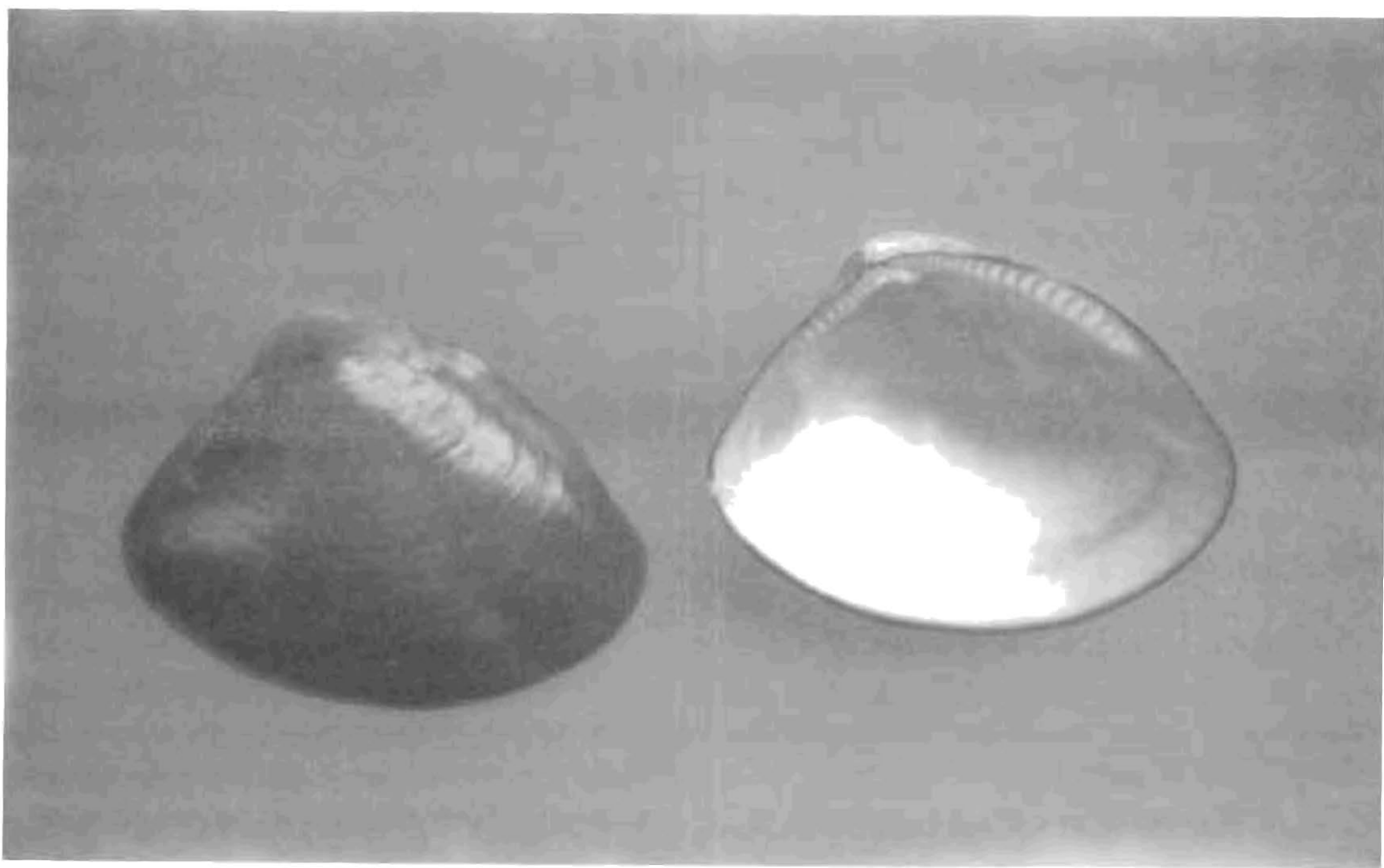

Figura 2. Ennucula cardara, longitud $17,7 \mathrm{~mm}$.

Comentarios: Maxwell (1988) reconoce a Ennucula Iredale, 1931 como un género para las especies actuales.

\section{Superfamilia NUCULANOIDEA}

Familia Nuculanidae H. Adams \& A. Adams, 1858

Nuculana (Nuculana) extenuata (Dall, 1897)

(Figura 3)

Leda extenuata Dall, 1897: 8, pl. 2, Fig. 2. Nuculana (Thestyleda) extenucia (Dall, 1897); Bernard, 1983: 13.

Nuculana (N.) extenuata (Dall, 1897); Coan et al., 2000: 85, pl. 6.

Nuculana (N) loshka (Dall, 1908); Keen, 1971: 31; Fig.34; Bernard, 1983: 13; Skoglund, 1991: 4.

Periostraco de color pardo y brillante: conchilla delgada con rostro alargado; con liraciones espaciadas que son más juntas y pro- minentes en el umbo; charnela con 19 dicntes anteriores y 31 posteriores. Longitud 17,0 $\mathrm{mm}$.

Distribución geográfica: Frente a Sitka, Alaska a Panamá, en 2000-2900 m (Coan et al., 2000).

Nueva localidad: $05^{\circ} 08,01^{\prime}$ S; $81^{\circ} 29,56^{\prime}$ W.

Hábitat: Fondo blando, a $864 \mathrm{~m}$.

Material examinado: 1 lote, 1 ejemplar, 23$07-96$.

Familia Yoldiidae Habe, 1977

Orthoyoldia panamensis (Dall, 1908)

(Figura 4)

Yoldia (Orthoyoldia) panamensis Dall, 1908: 219, 380; Keen, 197 1: 35. Fig. 59; Bernard, 1983: 14.

Orthoyoldia panamensis (Dall. 1908); Skoglund, 199!: 5.

Conchilla alargada translúcida, con finas líneas de crecimiento y los extremos rcdondeados; periostraco de color pardo y brillan- 
te; charnela con 24 dientes anteriores y 26 posteriores. Longitud $20,5 \mathrm{~mm}$.

Distribución geográfica: Bahía de Panamá, de 315 a 359 m (Keen, 1971).

\section{Nueva localidad:}

$$
\begin{aligned}
& 03^{\circ} 59,74^{\prime} \mathrm{S} ; 81^{\circ} 13,41^{\prime} \mathrm{W} \\
& 05^{\circ} 08,01^{\prime} \mathrm{S} ; 81^{\circ} 29,56^{\prime} \mathrm{W} \\
& 06^{\circ} 37,47^{\prime} \mathrm{S} ; 81^{\circ} 00,36^{\prime} \mathrm{W}
\end{aligned}
$$

Hábitat: Fondo blando, entre 400-864 m.

\section{Material examinado:}

1 lote, 1 ejemplar, 28-07-96

1 lote, 2 ejemplares, 23-07-96

1 lote, 6 ejemplares, 22-07-96

Comentarios: Bernard (1983) la cita para el Pleistoceno a los $07^{\circ} \mathrm{N}$.

\section{DISCUSIÓN}

Los Protobranchia constituyen el grupo más primitivo entre los bivalvos. Sin embargo presentan una mezcla de estructuras espe- cializadas (e. g. pie, ctenidios, palpos labiales) producto de una gran radiación adaptativa, tanto en las especies recientes (particularmente las formas abisales), como en las especies fósiles (Villarroel y Stuardo, 1998).

Se han reportado 18 especies de Protobranchia (Paredes et al., 1999a) para la costa Peruana, y recientemente ha sido citada por Coan et al. (2000) la especie Bathyspinula (B.) calcarella (Dall, 1908). Con la adición de las especies Nucula (Nucula) iphigenia Dall, 1908; Ennucula cardara (Dall, 1916); Nuculana (Nuculana) extenuata (Dall, 1897); Orthoyoldia panamensis (Dall, 1908), registradas en este trabajo, el número de bivalvos Protobranchia se incrementa a 23.

Los ejemplares de Orthoyoldia panamensis fueron obtenidos en profundidades mayores que la de los encontrados en la bibliografía consultada. En cambio los ejemplares de Ennucula cardara y Nuculana (N.) extenuata fueron obtenidos en profundidades menores.

En cuanto a la distribución temporal de las especies, Ennucula cardara y Nuculana (N.)

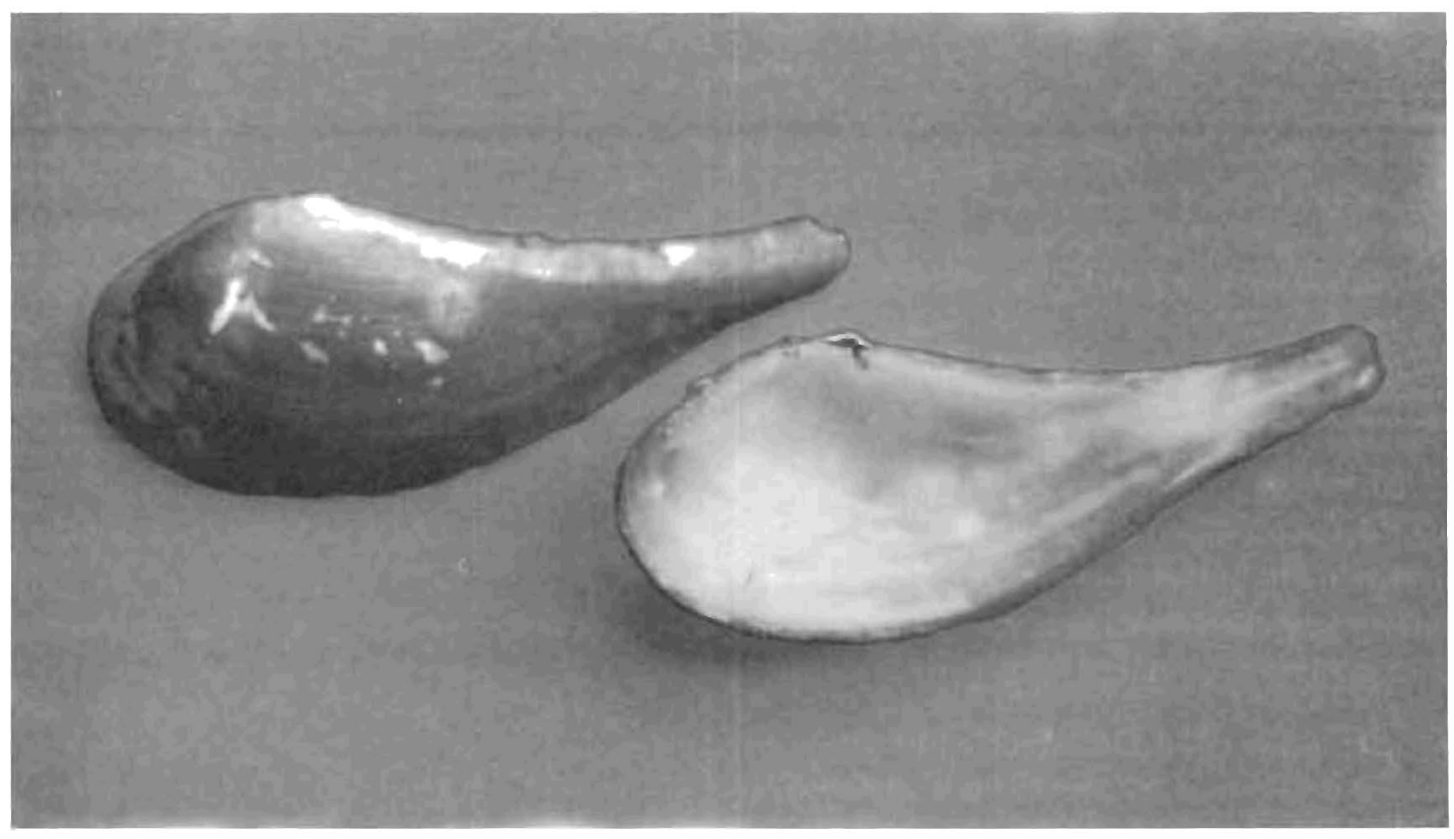

Figura 3. Nuculana (Nuculana) extenuata, longitud $17,0 \mathrm{~mm}$. 


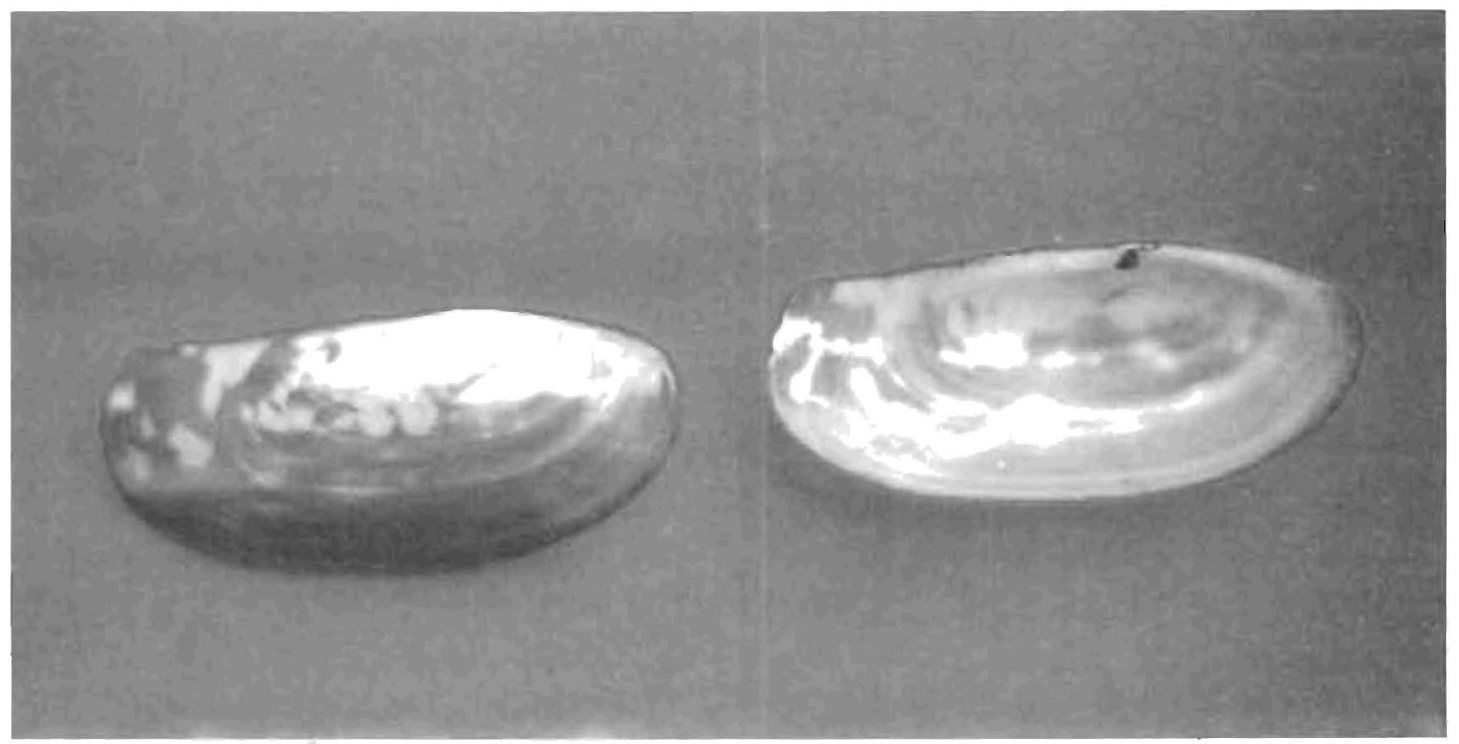

Figura 4. Orthoyoldia panamensis, longitud $16,7 \mathrm{~mm}$.

extenuata no presenta registros fósiles, por lo que se consideran especies recientes (Bernard, 1983; Coan et al. 2000). En cambio, Nucula (N.) iphigenia y Orthoyoldia pananensis según Bernard (1983), presentan registros fósiles desde el Pleistoceno.

\section{LITERATURA CITADA}

Bernard, F. R. 1983. Catalogue of the Living Bivalvia of the Eastern Pacific Ocean: Bering Strait to Cape Horn. Can. Spec. Publ. Fish. Aquat. Sci. 61: $1-102$.

Coan, E. V.; P. V. Scott \& F. R. Bernard. 2000. Bivalve Seashells of Westem North America: Marine Bivalve Mollusks from Arctic to Baja California. Santa Barbara Museum of Natural History Monographs Number 2. Studies in Biodiversity Number 2. USA 764 pp.

Dall, W. H. 1896. Diagnosis of new species of mollusks from the west coast of America. Natl. Mus. 18: 7-20.

Dall, W. H. 1897. Notice of some new or interesting species of shells from British Columbia and the adjacent region. Bull. Nat. Hist. Soc. British Columbia 2: $1-18$.

Dall, W. R. 1908. Reports on the dredging operations off the west coast of central America to the Galapagos, to the west coast of Mexico, and in the Gulf of California [...] XIV. The Mollusca and Brachiopoda. Bull, Mus. Comp. Zool. 43: 205-487.

Dall. W. H. 1916. Check list of the recent bivalve mollusks (Pelecypoda) of the Northwest coast of America from the Polar Sea to San Diego. California Southwest. Mus. Los Angeles, Calif: $1-44$.

Kameya, A.; R. Castillo; L. Escudero; E. Tello; V. Blaskovic; J. Córdova; Y. Hooker; M. Gutiérrez y S. Mayor, 1997. Localización, distribución y concentración de langostinos rojos de profundidad. Crucero BIC Humboldt 9607-08 (18 de julio a 06 de agosio de 1996). Pub. Esp. Inst. Mur Perú: 1-47.

Keen, A. M. 1971. Sea Shells of Tropical West America: Marine Mollusks from Baja California 10 Peru. $2^{\text {nd }} \mathrm{Ed}$. Stunford Univ. Press, California, $1064 \mathrm{pp}$.

Maxwell, P. A. 1988. Comments on "A reclassification of the Recent gen:ra of the subclass Protobranchia (Mollusca: Bivalvia)" by J. A. All.: and F. J. Hannah (1896). Jour. Conch. 33(2): 85-96.

Olsson, A. A. 1942. Tentiary and Quatemary fossils from the Burica Peninsula of Panama and Costa Rica. Bull. Am. Paleontol. 27:157-258.

Olsson, A. A. 1961. Mollusks of the Tropical eastern Pacific particularly from the south half of the Panamic-Pacific faunal province (Panama to Peru). Panamic-Pacific Pelecypoda. Am. 
Paleontol. Res. Inst. (Ithaca), 574 pp.

Paredes, C. 1974. Contribución al conocimiento de los invertebrados del litoral rocoso del Departamento de Lima con estudio especial de la biocenosis de mitílidos. Tesis doctoral. UNMSM. Lima, Perú. $141 \mathrm{pp}$.

Paredes, C. y F. Cardoso. 1999a. Estado actual del conocimiento de los moluscos acuáticos en el Perú. Rev. Peru. Biol. 6(1): 5-47.

Paredes, C.; F. Cardoso y J. Tarazona. 1999b. Invertebrados del intermareal rocoso del Departamento de Lima, Perú: Una lista comentada de especies. Rev. peru. Biol.. 6(1): 143-151.

Peña, M. 1971. Zonas de distribución de los bivalvos marinos del Perú. An. Ciènt. Univ. Nac. Agra- ria $9(1 / 2): 46-55$.

Skoglund, C. 1991. Additions to the Panamic Province Bivalve (Mollusca) literature 1971 to 1990 The Festivus 22 (Suppl. 2 to 1990): 1-74.

Valdivieso, V. 1984. Moluscos bivalvos del mar peruano. Boletín de Lima 34: 84-96.

Vegas-Vélez, M. 1968. Revisión taxonómica y zoogeográfica de algunos gasteropodos y lamelibranquios marinos del Perú. An. Cient. Univ. Nac. Agraria 6(1/2): 1-29.

Villarroel, M. y J. Stuardo. 1998. Protobranchia (Mollusca: Bivalvia) chilenos recientes y algunos fósiles. Malacologia 40(1/2): 113-229. 